$\mathrm{J}$ o u r n a l of

Mathematics

and Applications

JMA No 36, pp 85-93 (2013)

\title{
Some new generalized classes of difference sequences of fuzzy numbers defined by a sequence of Orlicz functions
}

\author{
Sunil K. Sharma
}

Submitted by: Marian Mattoka

\begin{abstract}
In the present paper we introduce some new generalized classes of difference sequence spaces of fuzzy numbers defined by a sequence of Orlicz functions. We also make an effort to study some topological properties and prove some inclusion relations between these spaces.
\end{abstract}

AMS Subject Classification: 40D05, 40A05, 46540

Keywords and Phrases: fuzzy numbers, Orlicz function, difference sequence spaces

\section{Introduction and Preliminaries}

Fuzzy set theory, compared to other mathematical theories, is perhaps the most easily adaptable theory to practice. The main reason is that a fuzzy set has the property of relativity, variability and inexactness in the definition of its elements. Instead of defining an entity in calculus by assuming that its role is exactly known, we can use fuzzy sets to define the same entity by allowing possible deviations and inexactness in its role. This representation suits well the uncertainties encountered in practical life, which make fuzzy sets a valuable mathematical tool. The concepts of fuzzy sets and fuzzy set operations were first introduced by Zadeh [11] and subsequently several authors have discussed various aspects of the theory and applications of fuzzy sets such as fuzzy topological spaces, similarity relations and fuzzy orderings, fuzzy measures of fuzzy events, fuzzy mathematical programming. Matloka [7] introduced bounded and convergent sequences of fuzzy numbers and studied some of their properties. For more details about sequence spaces of fuzzy numbers see ([1], [2], [3], [8], [10]) and references therein.

Let $C\left(\mathbb{R}^{n}\right)=\left\{A \subset \mathbb{R}^{n}: A\right.$ is compact and convex set $\}$. The space $C\left(\mathbb{R}^{n}\right)$ has a linear structure induced by the operations $A+B=\{a+b: a \in A, b \in B\}$ and

COPYRIGHT (C) by Publishing Department Rzeszów University of Technology P.O. Box 85, 35-959 Rzeszów, Poland 
$\lambda A=\{\lambda a: a \in A\}$ for $A, B \in C\left(\mathbb{R}^{n}\right)$ and $\lambda \in \mathbb{R}$.

The Hausdorff distance between $A$ and $B$ in $C\left(\mathbb{R}^{n}\right)$ is defined by

$$
\delta_{\infty}(A, B)=\max \left\{\sup _{a \in A} \inf _{b \in B}\|a-b\|, \sup _{b \in B} \inf _{a \in A}\|a-b\|\right\} .
$$

It is well known that $\left(C\left(\mathbb{R}^{n}\right), \delta_{\infty}\right)$ is a complete metric space.

A fuzzy number is a function $X$ from $\mathbb{R}^{n}$ to $[0,1]$ which is normal, fuzzy convex, upper semicontinuous and the closure of $\left\{X \in \mathbb{R}^{n}: X(x)>0\right\}$ is compact. These properties imply that for each $0<\alpha \leq 1$, the $\alpha$-level set

$$
X^{\alpha}=\left\{X \in \mathbb{R}^{n}: X(x)>\alpha\right\}
$$

is non-empty compact, convex subset of $\mathbb{R}^{n}$ with support $X^{0}$.

If $\mathbb{R}^{n}$ is replaced by $\mathbb{R}$, then obviously the set $C\left(\mathbb{R}^{n}\right)$ is reduced to the set of all closed bounded intervals $A=[\underline{A}, \bar{A}]$ on $\mathbb{R}$, and also

$$
\delta_{\infty}(A, B)=\max (|\underline{A}-\underline{B}|,|\bar{A}-\bar{B}|) .
$$

Let $L(\mathbb{R})$ denote the set of all fuzzy numbers. The linear structure of $L(\mathbb{R})$ induces the addition $X+Y$ and the scalar multiplication $\lambda X$ in terms of $\alpha$-level sets, by

$$
[X+Y]^{\alpha}=[X]^{\alpha}+[Y]^{\alpha}
$$

and

$$
[\lambda X]^{\alpha}=\lambda[X]^{\alpha}
$$

for each $0 \leq \alpha \leq 1$. The set $\mathbb{R}$ of real numbers can be embedded in $L(\mathbb{R})$ if we define $\bar{r} \in L(\mathbb{R})$ by

$$
\bar{r}(t)= \begin{cases}1, & \text { if } t=r \\ 0, & \text { if } t \neq r\end{cases}
$$

The additive identity and multiplicative identity of $L(\mathbb{R})$ are denoted by $\overline{0}$ and $\overline{1}$, respectively. For $r \in \mathbb{R}$ and $X \in L(\mathbb{R})$, the product $r X$ is defined as follows :

$$
r X(t)=\left\{\begin{array}{l}
X\left(r^{-1} t\right), \quad \text { if } \quad r \neq 0 \\
0, \quad \text { if } r=0
\end{array}\right.
$$

Define a map $d: L(\mathbb{R}) \times L(\mathbb{R}) \rightarrow \mathbb{R}$ by

$$
d(X, Y)=\sup _{0 \leq \alpha \leq 1} \delta_{\infty}\left(X^{\alpha}, Y^{\alpha}\right)
$$

For $X, Y \in L(\mathbb{R})$ define $X \leq Y$ if and only if $X^{\alpha} \leq Y^{\alpha}$ for any $\alpha \in[0,1]$. It is known that $(L(\mathbb{R}), d)$ is complete metric space (see $[7])$.

A sequence $X=\left(X_{k}\right)$ of fuzzy numbers is a function $X$ from the set $\mathbb{N}$ of natural numbers into $L(\mathbb{R})$. The fuzzy number $X_{k}$ denotes the value of the function at $k \in \mathbb{N}$. By $w(F)$ we denote the set of all sequences $X=\left(X_{k}\right)$ of fuzzy numbers. A sequence 
$X=\left(X_{k}\right)$ of fuzzy numbers is said to be bounded if the set $\left\{X_{k}: k \in \mathbb{N}\right\}$ of fuzzy numbers is bounded.

By $l_{\infty}(F)$ we denote the set of all bounded sequences $X=\left(X_{k}\right)$ of fuzzy numbers. A sequence $X=\left(X_{k}\right)$ of fuzzy numbers is said to be convergent to a fuzzy number $X_{0}$ if for every $\epsilon>0$ there is a positive integer $k_{0}$ such that $d\left(X_{k}, X_{0}\right)<\epsilon$ for $k>k_{0}$.

We denote by $c(F)$ the set of all convergent sequences $X=\left(X_{k}\right)$ of fuzzy numbers. It is straightforward to see that $c(F) \subset l_{\infty} \subset w(F)$.

Nanda [9] studied the classes of bounded and convergent sequences of fuzzy numbers and showed that these are complete metric spaces.

The notion of difference sequence spaces was introduced by Kizmaz [5], who studied the difference sequence spaces $l_{\infty}(\Delta), c(\Delta)$ and $c_{0}(\Delta)$. The notion was further generalized by Et and Çolak [4] by introducing the spaces $l_{\infty}\left(\Delta^{n}\right), c\left(\Delta^{n}\right)$ and $c_{0}\left(\Delta^{n}\right)$. Let $w$ be the space of all complex or real sequences $x=\left(x_{k}\right)$ and let $r$ be non-negative integer, then for $Z=l_{\infty}, c, c_{0}$ we have sequence spaces

$$
Z\left(\Delta^{r}\right)=\left\{x=\left(x_{k}\right) \in w:\left(\Delta^{r} x_{k}\right) \in Z\right\},
$$

where $\Delta^{r} x=\left(\Delta^{r} x_{k}\right)=\left(\Delta^{r-1} x_{k}-\Delta^{r-1} x_{k+1}\right)$ and $\Delta^{0} x_{k}=x_{k}$ for all $k \in \mathbb{N}$, which is equivalent to the following binomial representation

$$
\Delta^{r} x_{k}=\sum_{v=0}^{r}(-1)^{v}\left(\begin{array}{c}
r \\
v
\end{array}\right) x_{k+v} .
$$

Taking $r=1$, we get the spaces which were introduced and studied by Kizmaz [5].

An Orlicz function $M:[0, \infty) \rightarrow[0, \infty)$ is a continuous, non-decreasing and convex function such that $M(0)=0, M(x)>0$ for $x>0$ and $M(x) \longrightarrow \infty$ as $x \longrightarrow \infty$.

Lindenstrauss and Tzafriri [6] used the idea of Orlicz function to define the following sequence space,

$$
\ell_{M}=\left\{x \in w: \sum_{k=1}^{\infty} M\left(\frac{\left|x_{k}\right|}{\rho}\right)<\infty\right\}
$$

which is called as an Orlicz sequence space. Also $\ell_{M}$ is a Banach space with the norm

$$
\|x\|=\inf \left\{\rho>0: \sum_{k=1}^{\infty} M\left(\frac{\left|x_{k}\right|}{\rho}\right) \leq 1\right\}
$$

Also, it was shown in [6] that every Orlicz sequence space $\ell_{M}$ contains a subspace isomorphic to $\ell_{p}(p \geq 1)$. An Orlicz function $M$ satisfies $\Delta_{2}$-condition if and only if for any constant $L>1$ there exists a constant $K(L)$ such that $M(L u) \leq K(L) M(u)$ for all values of $u \geq 0$. An Orlicz function $M$ can always be represented in the following integral form

$$
M(x)=\int_{0}^{x} \eta(t) d t
$$

where $\eta$ is known as the kernel of $M$, is right differentiable for $t \geq 0, \eta(0)=0, \eta(t)>0$, $\eta$ is non-decreasing and $\eta(t) \rightarrow \infty$ as $t \rightarrow \infty$. 
The following inequality will be used throughout the paper. Let $p=\left(p_{k}\right)$ be a sequence of positive real numbers with $0<p_{k} \leq \sup _{k} p_{k}=H$ and let $K=\max \left\{1,2^{H-1}\right\}$. Then for sequences $\left\{a_{k}\right\}$ and $\left\{b_{k}\right\}$ in the complex plane, we have

$$
\left|a_{k}+b_{k}\right|^{p_{k}} \leq K\left(\left|a_{k}\right|^{p_{k}}+\left|b_{k}\right|^{p_{k}}\right) .
$$

Let $\sigma$ be a one-to-one mapping of the set of positive integers into itself such that $\sigma^{k}(n)=\sigma\left(\sigma^{k-1}(n)\right), k=1,2,3, \cdots$. Let $\mathcal{M}=\left(M_{k}\right)$ be a sequence of Orlicz function and $p=\left(p_{k}\right)$ be a bounded sequence of positive real numbers. We define the following classes of sequences of fuzzy numbers :

$$
\begin{aligned}
c_{0}^{F}\left(\mathcal{M}, \Delta^{r}, p, \sigma, s\right)= & \left\{X=\left(X_{k}\right) \in w^{F}: \lim _{k} \frac{1}{k^{s}}\left[M_{k}\left(\frac{d\left(\Delta^{r} X_{\sigma^{k}(n)}, \overline{0}\right)}{\rho}\right)\right]^{p_{k}}=0,\right. \\
& \text { uniformly in } n \text { for some } \rho>0, s \geq 0\}, \\
c^{F}\left(\mathcal{M}, \Delta^{r}, p, \sigma, s\right)= & \left\{X=\left(X_{k}\right) \in w^{F}: \lim _{k} \frac{1}{k^{s}}\left[M_{k}\left(\frac{d\left(\Delta^{r} X_{\sigma^{k}(n)}, X_{0}\right)}{\rho}\right)\right]^{p_{k}}=0,\right. \\
& \text { uniformly in } n \text { for some } \rho>0, s \geq 0\}
\end{aligned}
$$

and

$$
\begin{gathered}
l_{\infty}^{F}\left(\mathcal{M}, \Delta^{r}, p, \sigma, s\right)=\left\{X=\left(X_{k}\right) \in w^{F}: \lim _{k} \frac{1}{k^{s}}\left[M_{k}\left(\frac{d\left(\Delta^{r} X_{\sigma^{k}(n)}, \overline{0}\right)}{\rho}\right)\right]^{p_{k}}=0\right. \\
\text { for some } \rho>0, s \geq 0\} .
\end{gathered}
$$

If we take $\mathcal{M}(x)=x$, we get the spaces as follows

$$
\begin{aligned}
c_{0}^{F}\left(\Delta^{r}, p, \sigma, s\right)= & \left\{X=\left(X_{k}\right) \in w^{F}: \lim _{k} \frac{1}{k^{s}}\left(\frac{d\left(\Delta^{r} X_{\sigma^{k}(n)}, \overline{0}\right)}{\rho}\right)^{p_{k}}=0,\right. \\
& \text { uniformly in } n \text { for some } \rho>0, s \geq 0\}, \\
c^{F}\left(\Delta^{r}, p, \sigma, s\right)= & \left\{X=\left(X_{k}\right) \in w^{F}: \lim _{k} \frac{1}{k^{s}}\left(\frac{d\left(\Delta^{r} X_{\sigma^{k}(n)}, X_{0}\right)}{\rho}\right)^{p_{k}}=0,\right. \\
& \text { uniformly in } n \text { for some } \rho>0, s \geq 0\}
\end{aligned}
$$

and

$$
l_{\infty}^{F}\left(\Delta^{r}, p, \sigma, s\right)=\left\{X=\left(X_{k}\right) \in w^{F}: \lim _{k} \frac{1}{k^{s}}\left(\frac{d\left(\Delta^{r} X_{\sigma^{k}(n)}, \overline{0}\right)}{\rho}\right)^{p_{k}}=0,\right.
$$




$$
\text { for some } \rho>0, s \geq 0\} \text {. }
$$

If $p=\left(p_{k}\right)=1, \forall k$, we have

$$
\begin{aligned}
c_{0}^{F}\left(\mathcal{M}, \Delta^{r}, \sigma, s\right)= & \left\{X=\left(X_{k}\right) \in w^{F}: \lim _{k} \frac{1}{k^{s}} M_{k}\left(\frac{d\left(\Delta^{r} X_{\sigma^{k}(n)}, \overline{0}\right)}{\rho}\right)=0,\right. \\
& \text { uniformly in } n \text { for some } \rho>0, s \geq 0\}, \\
c^{F}\left(\mathcal{M}, \Delta^{r}, \sigma, s\right)= & \left\{X=\left(X_{k}\right) \in w^{F}: \lim _{k} \frac{1}{k^{s}} M_{k}\left(\frac{d\left(\Delta^{r} X_{\sigma^{k}(n)}, X_{0}\right)}{\rho}\right)=0,\right. \\
& \text { uniformly in } n \text { for some } \rho>0, s \geq 0\}
\end{aligned}
$$

and

$$
\begin{gathered}
l_{\infty}^{F}\left(\mathcal{M}, \Delta^{r}, \sigma, s\right)=\left\{X=\left(X_{k}\right) \in w^{F}: \lim _{k} \frac{1}{k^{s}} M_{k}\left(\frac{d\left(\Delta^{r} X_{\sigma^{k}(n)}, \overline{0}\right)}{\rho}\right)=0,\right. \\
\text { for some } \rho>0, s \geq 0\} .
\end{gathered}
$$

If we take $r, s=0, \sigma(n)=n+1, \mathcal{M}(x)=x$ and $p=\left(p_{k}\right)=1$ then we obtain the classes $c_{0}^{F}, c^{F}$ and $l_{\infty}^{F}$ of ordinary null, convergent and bounded sequences of fuzzy numbers, respectively which were defined and studied by Matloka [7].

The main purpose of this paper is to study some new generalized difference sequence spaces of fuzzy numbers defined by a sequence of Orlicz functions. We also examine some properties of these sequence spaces.

\section{Main results}

Proposition 2.1 If $d$ is a translation invariant metric on $L(\mathbb{R})$ then

(i) $(X+Y, \overline{0}) \leq \bar{d}(X, \overline{0})+\bar{d}(Y, \overline{0})$,

(ii) $d(\lambda X, \overline{0}) \leq|\lambda| d(X, \overline{0}),|\lambda|>1$.

Proof. It is easy to prove so we omit the details.

Theorem 2.2 Let $\mathcal{M}=\left(M_{k}\right)$ be a sequence of Orlicz functions, the spaces $c_{0}^{F}\left(\mathcal{M}, \Delta^{r}, p, \sigma, s\right), c^{F}\left(\mathcal{M}, \Delta^{r}, p, \sigma, s\right)$ and $l_{\infty}^{F}\left(\mathcal{M}, \Delta^{r}, p, \sigma, s\right)$ are closed under the operations of addition and scalar multiplication if $d$ is a translation invariant metric.

Proof. If $d$ is translation metric, then

$$
d\left(\Delta^{r}\left(X_{\sigma^{k}(n)}+Y_{\sigma^{k}(n)}\right), X_{0}+Y_{0}\right) \leq d\left(\Delta^{r} X_{\sigma^{k}(n)}, X_{0}\right)+d\left(\Delta^{r} Y_{\sigma^{k}(n)}, Y_{0}\right)
$$

and

$$
d\left(\lambda \Delta^{r} X_{\sigma^{k}(n)}, \lambda X_{0}\right) \leq|\lambda| d\left(\Delta^{r} X_{\sigma^{k}(n)}, X_{0}\right)
$$


where $\lambda$ is a scalar with $0<\lambda \leq 1$. It is easy to see that the spaces $c_{0}^{F}\left(\mathcal{M}, \Delta^{r}, p, \sigma, s\right)$, $c^{F}\left(\mathcal{M}, \Delta^{r}, p, \sigma, s\right)$ and $l_{\infty}^{F}\left(\mathcal{M}, \Delta^{r}, p, \sigma, s\right)$ are closed under the operations of addition and scalar multiplication.

Theorem 2.3 If $\mathcal{M}=\left(M_{k}\right)$ be a sequence of Orlicz functions, then

$$
c_{0}^{F}\left(\mathcal{M}, \Delta^{r}, p, \sigma, s\right) \subset c^{F}\left(\mathcal{M}, \Delta^{r}, p, \sigma, s\right) \subset l_{\infty}^{F}\left(\mathcal{M}, \Delta^{r}, p, \sigma, s\right) .
$$

Proof. The inclusion $c_{0}^{F}\left(\mathcal{M}, \Delta^{r}, p, \sigma, s\right) \subset c^{F}\left(\mathcal{M}, \Delta^{r}, p, \sigma, s\right)$ is obvious. We have only to show that $c^{F}\left(\mathcal{M}, \Delta^{r}, p, \sigma, s\right) \subset l_{\infty}^{F}\left(\mathcal{M}, \Delta^{r}, p, \sigma, s\right)$. For this by using triangle inequality, we have

$$
\begin{aligned}
\frac{1}{k^{s}}\left[M_{k}\left(\frac{d\left(\Delta^{r} X_{\sigma^{k}(n)}, \overline{0}\right)}{\rho}\right)\right]^{p_{k}} & \leq \frac{1}{k^{s}}\left[M_{k}\left(\frac{d\left(\Delta^{r} X_{\sigma^{k}(n)}, X_{0}\right)}{\rho}\right)\right]^{p_{k}} \\
& +\frac{1}{k^{s}}\left[M_{k}\left(\frac{d\left(X_{0}, \overline{0}\right)}{\rho}\right)\right]^{p_{k}} \\
& \leq \frac{1}{k^{s}}\left[M_{k}\left(\frac{d\left(\Delta^{r} X_{\sigma^{k}(n)}, X_{0}\right)}{\rho}\right)\right]^{p_{k}} \\
& +\max \left(1, \frac{1}{k^{s}}\left[M_{k}\left(\frac{\left|X_{0}\right|}{\rho}\right)\right]^{p_{k}}\right) .
\end{aligned}
$$

Thus $X=\left(X_{k}\right) \in c^{F}\left(\mathcal{M}, \Delta^{r}, p, \sigma, s\right)$ implies that $X=\left(X_{k}\right) \in l_{\infty}^{F}\left(\mathcal{M}, \Delta^{r}, p, \sigma, s\right)$. This completes the proof.

Theorem 2.4 If $\mathcal{M}=\left(M_{k}\right)$ be a sequence of Orlicz functions, then $c^{F}\left(\mathcal{M}, \Delta^{r}, p, \sigma, s\right)$ is a complete metric space under the metric

$$
d(X, Y)=\inf \left\{\rho>0: \sup _{n, k} \frac{1}{k^{s}}\left[M_{k}\left(\frac{d\left(\Delta^{r}\left(X_{\sigma^{k}(n)}-Y_{\sigma^{k}(n)}\right)\right)}{\rho}\right)\right]^{p_{k}} \leq 1\right\} .
$$

Proof. Let $X=\left(X_{k}\right), \quad Y=\left(Y_{k}\right) \in c^{F}\left(\mathcal{M}, \Delta^{r}, p, \sigma, s\right)$. Let $\left\{X^{(i)}\right\}$ be a Cauchy sequence in $c^{F}\left(\mathcal{M}, \Delta^{r}, p, \sigma, s\right)$. Then given any $\epsilon>0$ there exists a positive integer $N$ depending on $\epsilon$ such that $d\left(X^{(i)}, X^{(j)}\right)<\epsilon$, for all $n, m \geq N$. Hence

$$
\sup _{n, k} \frac{1}{k^{s}}\left[M_{k}\left(\frac{d\left(\Delta^{r} X_{\sigma^{k}(n)}^{(i)}-\Delta^{r} X_{\sigma^{k}(n)}^{(j)}\right)}{\rho}\right)\right]^{p_{k}}<\epsilon \quad \forall i, j \geq N .
$$

Consequently $\left\{X_{k}^{(i)}\right\}$ is a Cauchy sequence in the metric space $L(\mathbb{R})$. But $L(\mathbb{R})$ is complete. So, $X_{k}^{(i)} \rightarrow X_{k}$ as $i \rightarrow \infty$. Hence there exists a positive integer $n_{0}$ such that

$$
\sup _{n, k} \frac{1}{k^{s}}\left[M_{k}\left(\frac{d\left(\Delta^{r} X_{\sigma^{k}(n)}^{\left(n_{0}\right)}-\Delta^{r} X_{\sigma^{k}(n)}\right)}{\rho}\right)\right]^{p_{k}}<\epsilon \quad \forall n_{0} \geq N .
$$


This implies that $\left(X_{k}\right) \in c^{F}\left(\mathcal{M}, \Delta^{r}, p, \sigma, s\right)$. Hence $c^{F}\left(\mathcal{M}, \Delta^{r}, p, \sigma, s\right)$ is a complete metric space. This completes the proof

Theorem 2.5 If $\liminf \left(\frac{p_{k}}{q_{k}}\right)>0$, then $c^{F}\left(\mathcal{M}, \Delta^{r}, q, \sigma, s\right) \subset c^{F}\left(\mathcal{M}, \Delta^{r}, p, \sigma, s\right)$.

Proof. Suppose that $\liminf \left(\frac{p_{k}}{q_{k}}\right)>0$ holds and $X=\left(X_{k}\right) \in c^{F}\left(\mathcal{M}, \Delta^{r}, q, \sigma, s\right)$. Then there is $\beta>0$ such that $p_{k}>\beta q_{k}$ for large $k \in N$. Hence for large $k$

$$
\frac{1}{k^{s}}\left[M_{k}\left(\frac{d\left(\Delta^{r} X_{\sigma^{k}(n)}, X_{0}\right)}{\rho}\right)\right]^{p_{k}} \leq\left(\frac{1}{k^{s}}\left[M_{k}\left(\frac{d\left(\Delta^{r} X_{\sigma^{k}(n)}, X_{0}\right)}{\rho}\right)\right]^{q_{k}}\right)^{\beta} .
$$

Since

$$
\frac{1}{k^{s}}\left[M_{k}\left(\frac{d\left(\Delta^{r} X_{\sigma^{k}(n)}, X_{0}\right)}{\rho}\right)\right]^{q_{k}}<1
$$

for each $k, n$ and for some $\rho>0$. Hence $X=\left(X_{k}\right) \in c^{F}\left(\mathcal{M}, \Delta^{r}, p, \sigma, s\right)$.

Theorem 2.6 If $0<p_{k} \leq q_{k} \leq 1$, then $l_{\infty}^{F}\left(\mathcal{M}, \Delta^{r}, q, \sigma, s\right)$ is closed subset of $l_{\infty}^{F}\left(\mathcal{M}, \Delta^{r}, p, \sigma, s\right)$

Proof. Suppose that $0<p_{k} \leq q_{k} \leq 1$ holds and $X=\left(X_{k}\right) \in c^{F}\left(\mathcal{M}, \Delta^{r}, p, \sigma, s\right)$. Then there is a constant $L>1$ such that

$$
\frac{1}{k^{s}}\left[M_{k}\left(\frac{d\left(\Delta^{r} X_{\sigma^{k}(n)}, \overline{0}\right)}{\rho}\right)\right]^{q_{k}} \leq L
$$

for each $k, n$ and for some $\rho>0$. This implies that

$$
\frac{1}{k^{s}}\left[M_{k}\left(\frac{d\left(\Delta^{r} X_{\sigma^{k}(n)}, \overline{0}\right)}{\rho}\right)\right]^{p_{k}} \leq L
$$

for each $k$ and $n$. Hence $X=\left(X_{k}\right) \in l_{\infty}^{F}\left(\mathcal{M}, \Delta^{r}, p, \sigma, s\right)$. To show that $l_{\infty}^{F}\left(\mathcal{M}, \Delta^{r}, q, \sigma, s\right)$ is closed, suppose that $X^{i}=\left(X_{k}^{i}\right) \in l_{\infty}^{F}\left(\mathcal{M}, \Delta^{r}, q, \sigma, s\right), X^{i} \rightarrow X_{0}$ and $X_{0} \in l_{\infty}^{F}\left(\mathcal{M}, \Delta^{r}, p, \sigma, s\right)$. Then for every $\epsilon, 0<\epsilon<1$ there is $i_{0} \in N$ such that for all $k, n$ and for some $\rho>0$

$$
\frac{1}{k^{s}}\left[M_{k}\left(\frac{d\left(\Delta^{r}\left(X_{\sigma^{k}(n)}-X_{0}\right), \overline{0}\right)}{\rho}\right)\right]^{p_{k}}<\epsilon \text { for } i>i_{0}
$$

Now

$$
\begin{aligned}
\frac{1}{k^{s}} & {\left[M_{k}\left(\frac{d\left(\Delta^{r}\left(X_{\sigma^{k}(n)}-X_{0}\right), \overline{0}\right)}{\rho}\right)\right]^{q_{k}} } \\
& <\frac{1}{k^{s}}\left[M_{k}\left(\frac{d\left(\Delta^{r}\left(X_{\sigma^{k}(n)}-X_{0}\right), \overline{0}\right)}{\rho}\right)\right]^{p_{k}}<\epsilon \text { for } i>i_{0} .
\end{aligned}
$$

Therefore $X=\left(X_{k}\right) \in l_{\infty}^{F}\left(\mathcal{M}, \Delta^{r}, q, \sigma, s\right)$ i.e. $l_{\infty}^{F}\left(\mathcal{M}, \Delta^{r}, q, \sigma, s\right)$ is closed subset of $l_{\infty}^{F}\left(\mathcal{M}, \Delta^{r}, p, \sigma, s\right)$. 
Theorem 2.7 Let $0<h=\inf p_{k} \leq \sup p_{k}=H<\infty$. For any sequence of Orlicz function $\mathcal{M}=\left(M_{k}\right)$ which satisfies $\Delta_{2}$-condition, then

$$
c^{F}\left(\Delta^{r}, p, \sigma, s\right) \subset c^{F}\left(\mathcal{M}, \Delta^{r}, p, \sigma, s\right) .
$$

Proof. Let $X=\left(X_{k}\right) \in c^{F}\left(\Delta^{r}, p, \sigma, s\right)$, so that $\lim _{k} \frac{1}{k^{s}}\left[d\left(\Delta^{r} X_{\sigma^{k}(n)}, X_{0}\right)\right]^{p_{k}}=0$, uniformly in $n$. Let $\epsilon>0$ and choose $\delta$ with $0<\delta<1$ such that $M_{k}(t)<\epsilon$ for $0 \leq t \leq \delta$. We can write

$$
y_{k}=\frac{d\left(X_{\sigma^{k}(n)}, X_{0}\right)}{\rho} .
$$

We consider

$$
\sum_{\substack{y_{k} \leq \delta \\ k \in N}} \frac{1}{k^{s}}\left[M_{k}\left(y_{k}\right)\right]^{p_{k}}+\sum_{\substack{y_{k}>\delta \\ k \in N}} \frac{1}{k^{s}}\left[M_{k}\left(y_{k}\right)\right]^{p_{k}} .
$$

For $y_{k} \leq \delta$, we have

$$
\frac{1}{k^{s}}\left[M_{k}\left(y_{k}\right)\right]^{p_{k}}<\frac{1}{k^{s}} \max \left(\epsilon, \epsilon^{h}\right)
$$

by using the continuity of $\left(M_{k}\right)$. For $y_{k}>\delta$, we have

$$
y_{k}<\frac{y_{k}}{\delta}<1+\frac{y_{k}}{\delta}
$$

Since $\mathcal{M}=\left(M_{k}\right)$ is non-decreasing and convex, it follows that

$$
M_{k}\left(y_{k}\right)<M\left(1+\frac{y_{k}}{\delta}\right) \leq \frac{1}{2} M_{k}(2)+\frac{1}{2} M_{k}\left(\frac{2 y_{k}}{\delta}\right) .
$$

Since $\mathcal{M}=\left(M_{k}\right)$ satisfies $\Delta_{2}$-condition, we have

$$
M_{k}\left(y_{k}\right) \leq \frac{K}{2} \frac{y_{k}}{\delta} M_{k}(2)=K \frac{y_{k}}{\delta} M_{k}(2) .
$$

Thus we have

$$
\frac{1}{k^{s}}\left[M_{k}\left(y_{k}\right)\right]^{p_{k}} \leq \frac{1}{k^{s}} \max \left(1,\left[K M_{k}(2) \delta^{-1}\right]^{H}\left[y_{k}\right]^{p_{k}}\right) .
$$

This implies that

$$
\frac{1}{k^{s}}\left[M_{k}\left(y_{k}\right)\right]^{p_{k}} \leq \frac{1}{k^{s}} \max \left(\epsilon, \epsilon^{h}\right) \frac{1}{k^{s}} \max \left(1,\left[K M_{k}(2) \delta^{-1}\right]^{H}\left[y_{k}\right]^{p_{k}}\right) .
$$

Taking $\epsilon \rightarrow 0$ and $k \rightarrow \infty$, it follows that $X=\left(X_{k}\right) \in c^{F}\left(\mathcal{M}, \Delta^{r}, p, \sigma, s\right)$.

Theorem 2.8 Let $\mathcal{M}, \mathcal{M}^{\prime}$ and $\mathcal{M}^{\prime \prime}$ are sequences of Orlicz functions. Then

(i) $Z\left(\mathcal{M}^{\prime}, \Delta^{r}, p, \sigma, s\right) \subset Z\left(\mathcal{M} \cdot \mathcal{M}^{\prime}, \Delta^{r}, p, \sigma, s\right)$;

(ii) $Z\left(\mathcal{M}^{\prime}, \Delta^{r}, p, \sigma, s\right) \cap Z\left(\mathcal{M}^{\prime \prime}, \Delta^{r}, p, \sigma, s\right) \subset Z\left(\mathcal{M}^{\prime}+\mathcal{M}^{\prime \prime}, \Delta^{r}, p, \sigma, s\right)$, where $Z=$ $c_{0}^{F}, c^{F}, l_{\infty}^{F}$.

Proof. It is easy to prove so we omit the details. 
Some new generalized classes of difference sequences of fuzzy numbers ...

\section{References}

[1] R. Çolak, Y. Altın and M. Mursaleen, On some sets of difference sequences of fuzzy numbers, Soft Computing, 15 (2011), 787-793.

[2] P. Diamond and P. Kloeden, Metric spaces of fuzzy sets, Fuzzy Sets Systems, 35 (1990), 241-249.

[3] A. Esi, On some new paranormed sequence spaces of fuzzy numbers defined by Orlicz function and statistical convergence, Math. Model. Anal., 11 (2006), 379388.

[4] M. Et and R. Çolak, On some generalized difference sequence spaces and related matrix transformations, Hokkaido Math. J., 26 (1997), 483-492.

[5] H. Kızmaz, On certain sequence spaces, Can. Math. Bull., 24 (1981), 169-176.

[6] J. Lindenstrauss and L. Tzafriri, On Orlicz sequence spaces, Israel J. Math., 10 (1971), 345-355.

[7] M. Matloka, Sequences of fuzzy numbers, BUSEFAL, 28 (1986), 28-37.

[8] M. Mursaleen and M. Başarır, On some new sequence spaces of fuzzy numbers, Indian J. Pure Appl. Math., 34 (2003), 1351-1357.

[9] S. Nanda, On sequences of fuzzy numbers, Fuzzy Sets and Systems, 33 (1989), 123-126.

[10] F. Nuray and E. Savaş, Statistical convergence of sequences of fuzzy numbers, Math. Slovaca, 45(3) (1995), 269-273.

[11] L. A. Zadeh, Fuzzy sets, Information and control, 8 (1965), 338-353.

DOI: $10.7862 / \mathrm{rf.2013.8}$

Sunil K. Sharma

email: sunilksharma42@yahoo.co.in

School of Mathematics

Shri Mata Vaishno Devi University

Katra-182320, J\&K, INDIA

Received 07.05.2013, Revisted 20.10.2013, Accepted 25.10.2013 